\title{
Successful Methods for Developing Introductory Design Labs As Preparation for Upper Year Discipline Selection and Multi-Disciplinary Design
}

\author{
T.C. Muench, P.Eng., \\ Associate Professor of Engineering \\ Listwin Family Chair in Innovative Teaching \\ University of Saskatchewan \\ tim.muench@usask.ca
}

\begin{abstract}
Introductory design labs accomplish important learning objectives in engineering education. The model used incorporates representative design labs from each discipline within the college. This allows students to learn the design process, gain exposure to each discipline, gain terminology and concepts to begin functioning in a multi-disciplinary design environment, and make a more informed choice of their career discipline.

This paper investigates, with examples and student feedback, several successful methods for developing freshman design labs, including:

Industry partnerships to bring professional practice into the classroom through real designs rolled back and then constrained.

- Adapting / simplifying upper year design labs by applying additional constraints.

- Simplified versions of upper year content that convey concepts and procedures without in-depth technical knowledge.
\end{abstract}

\section{Introduction}

The mandate of the Listwin Family Chair in Innovative Teaching includes engaging students in active participation and discussion of technical concepts; participating in, and leading, junior courses that introduce the unique process of engineering problem solving to all engineering students; and being a liaison with the engineering profession to help bring real engineering problems into the classroom and foster keen interest and enthusiasm for problem solving. The Listwin Family Chair was established by a personal donation of $\$ 2.5$ million from Don Listwin, a graduate of the college

Creating exciting, hands-on, practice-based labs is core to the mandate of the chair, and is a key element of the Introduction to Engineering II course that is coordinated by the Chairholder.

The design labs are created in a manner to reinforce the engineering design cycle [1]. Depending on the lab, various portions of the design cycle are constrained to limit the complexity and time commitment for the lab.

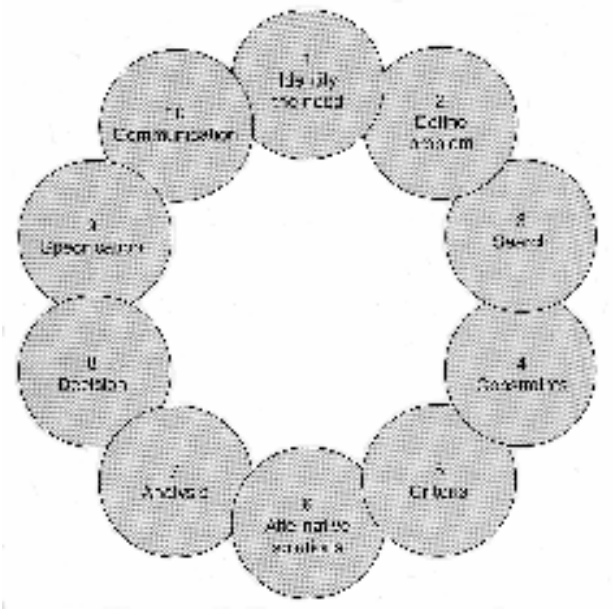

Figure 1 - Engineering Design Cycle

Whenever possible, the engineering design cycle is also used by faculty as a template for developing the lab content, and lab handouts are developed to require students to follow the engineering design cycle.

The time allotted to each of the labs is a 20 minute overview of the discipline (given by the department), followed by a 30 minute lecture that gives an overview of the lab. The actual lab period is a 3 hour period the following week, with access to a computer lab equipped with CAD and engineering software.

Section 1 provides an introduction and overview of the paper. Section 2 examines an industry partnership lab which is developed from a real design that is rolled back to a partially complete state, and then constrained to control the complexity. Section 3 examines the adaptation of an upper year design lab that has been 
simplified by adding additional constraints. Section 4 examines a lab that was produced by taking upper year content and providing simplified theory and basic technical background to allow the modification of a system to perform a new function.

\section{Industry Partnership - Design Rollback}

This section contains a summary and some excerpts from a separate work by the author [2], as well as discussion as to its effectiveness in this context. The lab to be designed in this case must be representative of the Mechanical discipline, be exciting, practicebased, use engineering analysis tools if possible, and be suitable for the students to implement the traditional engineering deign cycle.

\subsection{Securing a Good Industry Partner}

The first step in securing an industry partner is locating a well-placed contact. The best contacts are often graduates of the college, or someone within a company that has ties to the college, or both. This provides a receptive and motivated partner that will work with you to identify a project, extract the necessary design data and files, sanitize or get the necessary clearances for any data that has to be used, and answer your questions.

The industry partner selected for developing this lab was a local mechanical engineering design consulting firm, Loken Engineering, where the contact is the president, David Loken, who is also a graduate of the college. The company was the principle design consultant for one of the beamlines for the Canadian Light Source (CLS) Synchrotron, which was recently commissioned on the $\mathrm{U}$ of $\mathrm{S}$ campus.

\subsection{Overview of the Project}

The CLS synchrotron [3], shown schematically in Figure 2 is a football field-sized facility that produces light (principally X-rays - but millions of times more intense than conventional X-rays) with special qualities such as extreme brightness and short wavelengths.

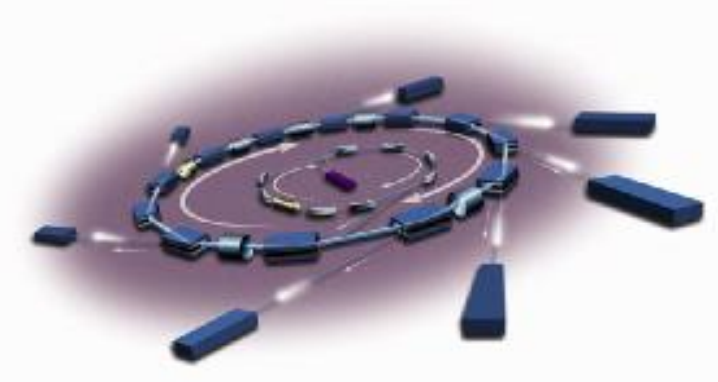

Figure 2 - Schematic of Synchrotron
The brilliant light allows matter to be 'seen' at the atomic scale, allowing for state-of-the-art investigations in materials science, medicine, biology, chemistry, physics, and the environmental sciences. Each beamline is designed for the type of light required, and it manipulates, filters and focuses the light to obtain the characteristics required by that particular beamline.

The selected project is a mask for mirror M1, shown in Figure 3 below, which mechanically blocks some of the high energy light, to protect the leading edges of the downstream mirror, M1. Mirrors within the beamline shape and focus the beam as part of the process of producing specific lighting characteristics.

The project involves aspects of heat transfer, fluid flow, Finite Element Analysis (FEA), 3-Dimensional CAD (Parametric Solids Modeling) and engineering approximation, all of which are representative of the Mechanical discipline.

It also contains application of theory, calculations, and a component of design that can be reasonably accomplished in the amount of time allotted to the students for the lab.

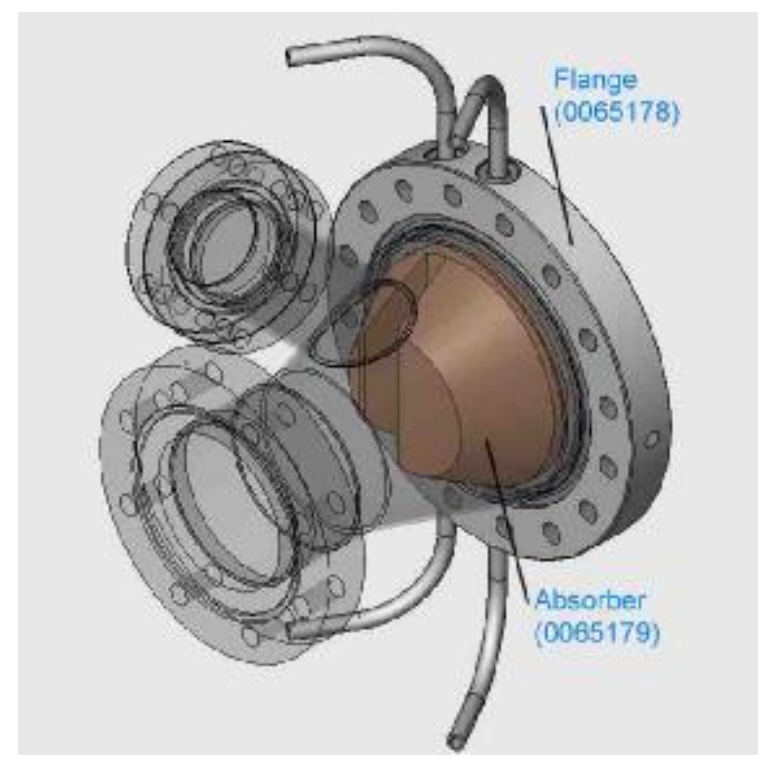

Figure 3 - M1 Mask and Cooling lines

A well documented design makes an excellent starting point for this type of lab. Design data, reference material, and CAD/FEA models for which permission to distribute was easy to obtain, were available to use. In this case, the research community associated with the CLS is very open, so the use of real data was not an issue. This minimized the resource investment by both the industry partner and the faculty member. In other instances, slight modifications or sanitization of data, with the 
permission of a client, can often make realistic data and models available.

In general, reducing the degrees of freedom of a real project to one or two can provide an excellent design project for a first year design course.

\subsection{Lab Specification}

It is important to look at the lab in terms of its ability to teach the engineering design cycle. The following sections show how each of the components of the design cycle were addressed in the design of the lab handout.

\subsubsection{Identification of Need}

The function of the M1 Mask is to block the excess light from striking the leading edge of the M1 mirror, and the need is to keep it within the allowable temperature specification.

\subsubsection{Problem Definition}

The heat generated by the impact of the intense light, and the inability of the ultra-high vacuum within the beamline chamber to dissipate the heat requires additional cooling. The problem is to determine the amount of cooling and how it should be implemented.

\subsubsection{Search}

The lab requires fluid flow and heat transfer theory that is beyond the current theoretical background of the students. The equations and related information were included in the main body of the lab, and further explanation, including the conditions under which they are valid, were included in an appendix.

\subsubsection{Constraints}

The constraints were provided in the lab handout, but were not specifically identified as such. These included the lack of heat convection, the light distribution of the synchrotron beam, worst-case cooling water temperature, ambient temperature, and the existing design and materials for the mask/flange.

\subsubsection{Criteria}

Exceeding the allowable temperature for the mask risks the emission of unwanted atomic particles into the ultra-high vacuum of the synchrotron. Also, if the velocity of cooling water exceeds the recommended range, the turbulent flow risks inducing vibration, which can damage the microscopic experiments.

Students were referred to an actual technical specification document for the Synchrotron design [4], and were required to glean the maximum allowable temperature. They were also required to locate the recommended operating range for cooling water velocity.

\subsubsection{Alternate Solutions}

The options were limited to 2 sets of potential calculations. The first represents laminar flow, which has extremely low risk of inducing experimentdamaging vibration in the extremely precise beamlines, but which is less effective at creating good convective heat transfer. The second option uses turbulent flow, which produces much better convective heat transfer, but which, at its higher flow rates, can induce unwanted and experiment damaging vibration.

\subsubsection{Analysis}

Design skills that often emerge with experience, and therefore are usually lacking in first year students, are rules of thumb and engineering approximation.

Because of the limitations of entering the heat from the synchrotron light into the FEA software, the actual 3-Dimensional light intensity distribution is approximated by 3 rectangular box shapes as shown in Figure 4. The basic shape of the approximation was given and explained, but the students were required to obtain values from the actual data specification for the synchrotron, and to extract the 3 equivalent power levels.

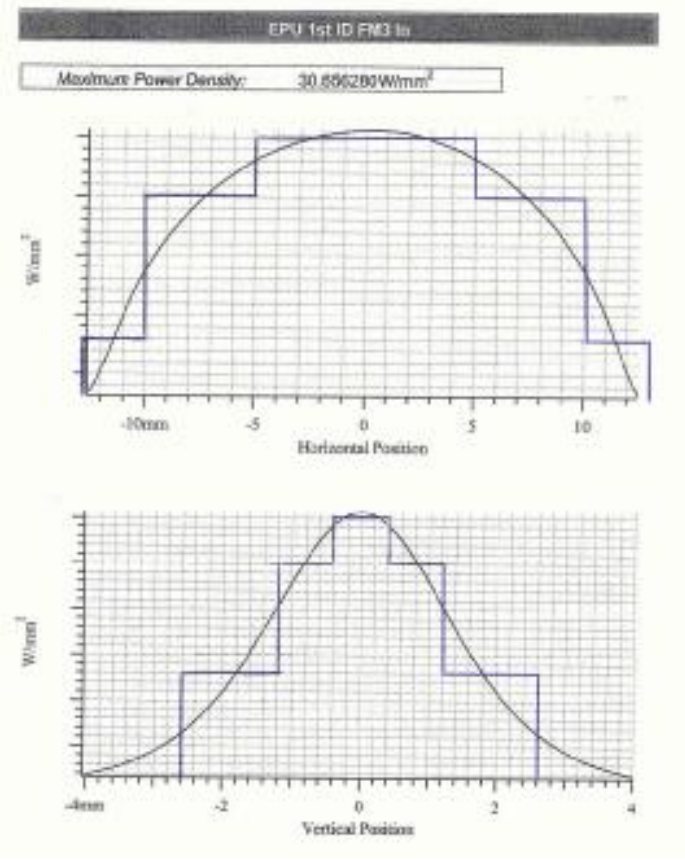

\section{Figure 4 - Approximation of Light Intensity}

The lab analysis involved leading the students through the first iteration of the FEA analysis. This involved setting and changing parameters, meshing the model, and running the analysis. The initial results are shown in Figure 5. 
Between iterations, new convection values were required for the cooling water, so the students used the appropriate laminar or turbulent flow equations to obtain the new convection coefficients.

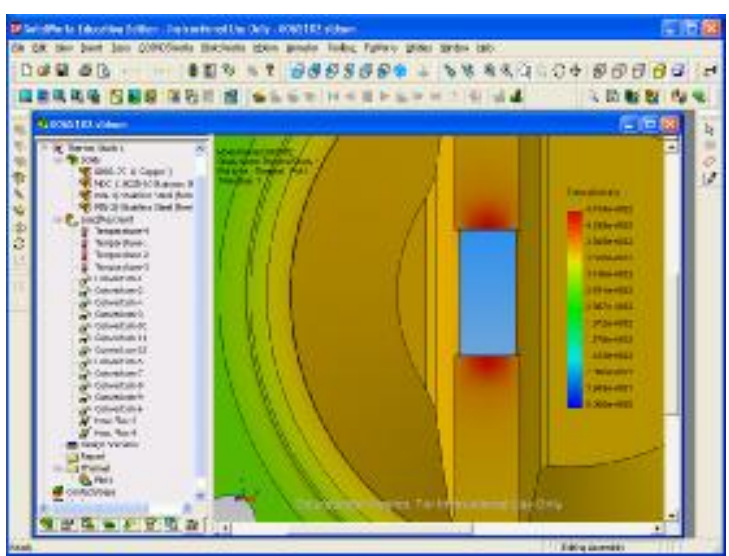

Figure 5 - Temperature Distribution

\subsubsection{Decision}

The final decision required was simply to select the type of flow rate that produced the cooling necessary to keep the temperature within specification, while ensuring that the velocity of the cooling water was within the recommended range so as not to induce vibration from the turbulence.

\subsubsection{Specification}

The volumetric flow rate and the temperature change of the cooling water were specified as results.

\subsubsection{Communication}

A full lab report, with calculations and analysis results was specified as a requirement.

\subsection{Summary and Feedback}

Implementation of the lab went smoothly, and feedback from students was very positive. A number of key components are responsible for the success.

\subsubsection{Lab Design}

Securing a good industry partner is key to the success. A well placed contact, and the ability to obtain release of design data, or at least sanitizing/modifying data to obtain permission to use it is also required.

The use of a methodical design process (the Engineering Design Cycle) will greatly increase the probability that a well designed lab will result.

Concurrent consideration of the students' requirement to follow the engineering design process will ensure that the lab lends itself to the design process.

\subsubsection{Lab Communication}

Communication can have a huge effect on the success of the lab. Any missing, unclear or confusing information, or an unintentional problem not core to the design lab, can torpedo an otherwise excellent lab.

\subsubsection{Training of Lab Personnel}

All faculty and Teaching Assistants involved with lab presentation and supervision must be familiar with lab and any basic software tools that they will be expected to troubleshoot in the lab.

The Introduction to Engineering II course uses faculty and TAs from various disciplines, so there is usually diversity, and sometimes a total lack, of background theory. As a result, a dry-run of the lab with faculty and TAs also proves to be an excellent step in the Quality Assurance process for the lab.

It should be noted that the training of lab personnel should be done with enough lead time that any errors or omissions that are uncovered can be corrected in time for the actual lab presentation.

\subsubsection{Survey Results}

The following survey results show the very positive results of the student feedback.

From the sample of over 200 respondents, more than 19 out of 20 agreed that:

the lab content was practical and related to a real-life engineering problem

Approximately $\mathbf{8}$ out of $\mathbf{1 0}$ of the respondents also agreed that:

the lab increased their interest and excitement for engineering design and

the lab was useful in expanding their engineering and design knowledge

\subsubsection{Further details}

Additional details on the development of the lab can be obtained from the original full paper, by contacting the author.

\section{Upper Year Lab - Modify/Constrain}

The approach used for this lab was to take an existing lab from an upper year course, and add additional constraints to limit the required knowledge, and limit the scope. The lab to be designed in this case must be representative of the Geological Engineering discipline, be realistic and practical, have a reasonable and clearly defined design component, and be suitable 
for the students to implement the traditional engineering deign cycle.

\subsection{Selecting a Representative Lab}

The design lab is based on a more advanced assignment used in the fourth year Petroleum Geomechanics class, and was designed by Dr. Chris Hawkes, Department of Geological Engineering. The intent of the lab is to design, select and specify well casing and couplings, as well as drilling mud density, for various stages of an oil well. Some of the following information has been excerpted or summarized from the lab materials produced by Dr. Hawkes [5].

\subsection{Lab Simplification}

In the full version of the assignment, two key elements are required for the casing design:

1. The selection of depths where each of the casing strings must be set, and

2. The selections of casing materials and couplings, as shown in Figure 6, based on attaining the required axial strength while minimizing cost.

This sounds straightforward, but it proves to be challenging for the fourth-year students because there are several different acceptable answers, depending on the approach used to solve the problem. This type of ambiguity, though essential for senior classes to grapple with, is not considered helpful in a first-year laboratory.

For the modified lab, the depths at which casing strings must be set are given, as are the diameters of each of the casings, so the students are only required to perform element number 2.

In addition, the most important performance properties of casing include its rated values for axial tension, burst pressure and collapse load. For this lab, only the axial tension on the casing will be used when selecting casing.

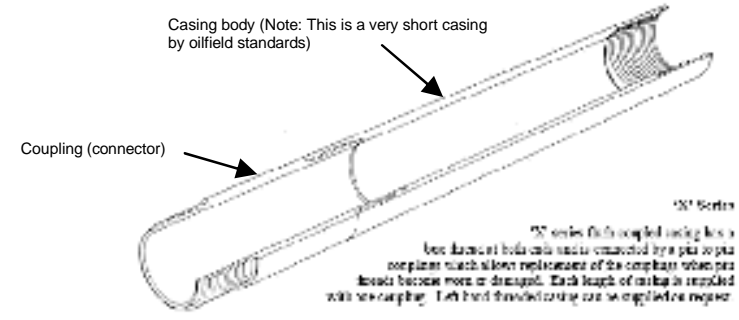

Figure 6 - Short Casing and Coupling

\subsection{Overview of the Lab}

When a casing string has reached its maximum depth (length), just before it is set by cementing it in place, it is essentially being suspended from the drill rig floor. At this point, the tensile load is most critical in the top joint of the string. The effective weight of the casing string can be calculated from the absolute weight of the casing (provided by the casing manufacturer), minus the buoyant force acting on the casing due to the mud that it is displacing.

The equation for the effective weight $W_{\text {eff }}$ (absolute weight minus the buoyant force) of a casing string of length $z$ with an absolute weight $W_{a b s}$, an outer diameter denoted by $O D$ and an inner diameter denoted by $I D$, if it is suspended in a drilling mud of density $\rho_{\text {mud }}$ is given in (1).

$$
w_{e f f}=w_{a b s}-\frac{\rho_{m u d} g \pi}{4}\left(O D^{2}-I D^{2}\right) \times z
$$

\subsubsection{Mud Density}

Figure 7 shows the depth profile of interpreted formation pore pressure and fracture pressure at the location where plans are being made to drill a vertical well to exploit an oil reservoir. If the drilling fluid density is less than the formation pore pressure, formation fluids may flow into the well, causing a blowout, and/or rocks may slough off of the walls of the borehole, resulting in borehole collapse conditions. Conversely, if the drilling fluid density exceeds the fracture gradient, tensile fractures may be created on the borehole wall and the drilling fluid will be lost into the formation.

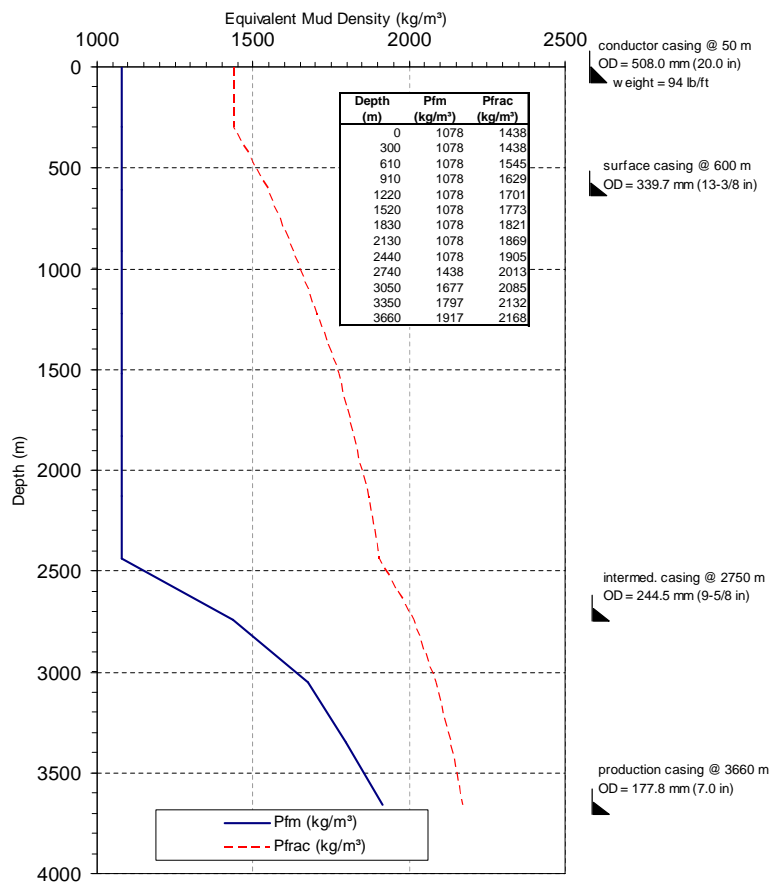

Figure 7 - Pore/fracture pressures

It is normal practice to drill with a drilling fluid density that is about $50 \mathrm{~kg} / \mathrm{m}^{3}$ greater than the formation pore pressure. If a depth is reached where the density required to achieve this condition exceeds 
the fracture pressure at any point in the well that is exposed to the drilling fluid, it is necessary to run a casing string into the well before drilling any further. Based on these considerations, the casing depths indicated in Figure 7 have been selected by the well design team.

\subsubsection{Design Steps} lab.

The following deliverables were specified for the

(a) Decide what mud densities to use while drilling this well. Use a coloured marker or pencil to draw the recommended mud density on Figure 7.

(b) For each casing string (except the conductor casing - no calculations are required for this string), specify the wall thickness, steel grade and thread type to avoid tensile yield. For these calculations, assume a maximum incremental hook load of $222 \mathrm{kN}(50,000 \mathrm{lbf})$ may be applied when pulling on stuck casing.

(c) Select a bit size to be used for each of the hole sections to be drilled (excluding the conductor casing). Select these properties such that the drill bit size for each interval is less than or equal to the drift diameter of the casing string that was selected for the preceding hole section. A table of commonly available bit sizes was provided.

Comments:

- For this well design exercise, only consider tensile yield design criteria. Burst and collapse will be neglected.

- For a given casing OD, the different casing types are ordered such that the cheapest grades of steel are listed first (i.e., H-40 is the cheapest, and P110 is the most costly). Start at the first row listed for each casing size in the provided table [6], and increment progressively downwards until you find a casing type that fulfills your design criteria.

\subsection{Lab Handouts}

A number of handouts with information relevant to the lab were provided.

\subsubsection{Discipline Overview}

As an introduction to the lab, a discipline overview, with an abundance of photos and graphics, and a lab overview provided a clear picture of the lab content.

\subsubsection{Lab Handout}

In order for the lab to be successful, the lab handout must make the lab concept and procedure absolutely clear. The lab handout shows sample calculations, the pore pressure plot, and casing and thread examples.

\subsubsection{Casing/Coupling table}

A table of properties for casing/couplings was provided to allow checks for tensile strength of casings and couplings, and volume calculations required to determine the buoyant force (due to mud).

\subsubsection{Drill Bit Table}

Drill bit sizes were one of the deliverables, so the table with relevant information was provided.

\subsection{Summary and Feedback}

Implementation of this lab again went smoothly, and feedback from students was again very positive. A number of key components are responsible for the success.

\subsubsection{Lab Simplification}

Constraining the degrees of freedom to make a lab manageable can still allow enough design freedom to make the lab enjoyable. The multi-step selection required for the casing design provided a reasonable challenge for students.

\subsubsection{Lab Communication}

Communication can have a huge effect on the success of the lab. As indicated in the previous lab, any missing, unclear or confusing information, or an unintentional problem not core to the design lab, can ruin an otherwise excellent lab.

\subsubsection{Survey Results}

The following survey results show the very positive results of the student feedback.

From the sample of over 200 respondents, approximately 9 out of 10 agreed that:

they found the lab to be realistic and practical

the lab had a reasonable and clearly defined design component

the lab gave them a better understanding of the Geological Engineering discipline

The responses show that the lab was overwhelmingly positively received.

\section{Simplify Upper Year Content}

The third method examined for developing introductory labs is to take representative theory and/or content from an upper year in the discipline, and simplify and constrain its presentation so that first year students can capably perform the design.

The following section contains summary information and some excerpts from another paper by 
the author, presented at CDEN 2005, as well as a discussion on the effectiveness of this method.

\subsection{Determining a Content Area}

This paper looks at the development of an Electrical Engineering lab. With only 2 hours of pre-lab lecture/preparation, and 3 hours in-lab time, providing the necessary theory and completing a meaningful lab is extremely challenging.

The existing electrical engineering discipline lab was entirely theoretical and calculation-based, and the department wanted a new hands-on lab that was represented of Electrical Engineering, and of the new Computer Engineering option that is in the final stages of approval.

A number of options were discussed between the author and the designated representative from the Department of Electrical Engineering, Dr. Eric Salt. It was determined that a microprocessor-based lab would be undertaken, but significant consideration would have to be given to simplifying the theory associated with it. Because of the promotional aspect for the department of Electrical Engineering, the department agreed to purchase the boards and supply the faculty resources to develop the lab.

The initial plan was to develop a monitor that would run and allow a simplified instruction set, but the high degree of customization and the low ability for it to be adaptable to new applications in the future, changed the focus. A reusable core of routines was developed for initialization, input/output and display, and the actual lab was implemented in a relatively small main program. Future lab applications can thus efficiently use the existing core of routines.

More than 2 weeks of full-time effort was required to produce a workable lab and lab handout [7]. The actual Lab handout is NOT discussed here, but the contents of it are described in 4.3.2 Lab Lecture section that follows.

\subsection{Overview of the Project}

With the limited time allowed for background preparation, and in-lab programming, creating a complete lab from scratch was not feasible. The project selected was based on the concept of providing an operational microprocessor system that had to be modified to perform a new function.

\subsubsection{Starting Point Provided - Keyless Entry}

The lab was designed such that students were given a working copy of the code that implemented a simulated keyless garage door opener based on a microprocessor board with a hexadecimal keypad and 4-digit display. The user types in the combination on the keypad, and when the correct combination is typed, the word OPEn is shown temporarily on the display.

\subsubsection{New Design - Hotel Room Door Lock}

A description of the operation of a hotel door room lock was provided. These locks are battery powered and have no electronic connection to the computer at the front desk. Real locks use magnetic strips, but the lab uses a keyed-in combination instead.

The hotel door lock recognizes 2 combinations. One is called the current-occupant combination, and the other is called the next-occupant combination. The next-occupant combination is related to the currentoccupant combination by an algorithm. In reality, this would be a complicated algorithm. The lab, however, will simply add 4 to the current-occupant combination to create the next-occupant combination.

When a new guest checks into the hotel the clerk gives the new guest the next-occupant combination. This is possible because the clerk's PC (the computer at the front desk) keeps track of the current-occupant combination for each room, so it can calculate the next-occupant combination using the same algorithm as the locks.

When the new guest opens the door with the nextoccupant combination for the first time, the lock changes both combinations. The current-occupant combination is changed to that of the next-occupant combination and a new next-occupant combination is created by the algorithm.

The combination used by the guest that checked out will not work once the next guest has opened the door (with the next-occupant combination). This also means the new guest becomes the current occupant upon first entry into the room.

In a real system, other information, such as scheduled checkout time, and combinations for housekeeping staff, may also be contained on the magnetic key. The scheduled checkout time can be used to disable the key (current-occupant combination) even if the next-occupant has not used their key yet.

The new design requires students to make the necessary modifications of the logic and program code to handle an additional combination, and a rolling combination, and then to design and implement the correct program flow to handle the current-occupant / new-occupant combinations.

\subsection{Theory/Background Preparation}

Students were assumed to have no electrical or computer programming background prior to the lab period. As a result, the background theory lecture and the lab lecture must supply all relevant information. 


\subsubsection{Background Theory Lecture}

When lab content is representative of upper year courses, even if the theory has been simplified, at least a conversational knowledge and some basic theory must be provided so that the students can be expected to modify an existing design.

The background lecture, entitled 'Introduction to Digital Computing' [8] was prepared by Dr. Hugh Wood, former Head of Electrical Engineering, AND currently Chief Operating Officer for VCOM (leading designer/manufacturer of products for Cable Television, Data over Cable, Digital Video, and Wireless Telecom), AND currently a Sessional Lecturer for Introduction to Engineering II.

The content of the lecture included:

- The pervasive nature of digital computing in all areas of engineering.

- Logic, including examples of button pressing on a calculator, and process control

- Flow charts, with an example for the calculator

- Number systems, so that students could understand the binary filter used in the lab

Decimal and hexadecimal addition, and their similar procedures

- Binary arithmetic and its link to hardware, microprocessors, and 'Exclusive Or' logic gates

- Field Effect Transistor (FET) for solid state switching, and combination with resistors to create logic gates

- More complex circuits from logic gates:

Adder

Memory (dynamic) - cheap

Flip-flops - a form of static memory

Latches - clocked flip-flops

Registers - made from several latches

Shift Registers - serial communication, or simultaneous transfer for a data bus

- Arranging components (registers, bus, adder etc.) into a processor

- Program instructions and their operation

- Input and output - keypad and display for lab

- Compiler and the use of meaningful names for instructions

Observation of the lecture showed that a small number of students were not paying close/any attention during the lecture. This seems to correlate with the small number of students who indicated on the feedback survey that they didn't have enough background for the lab.

\subsubsection{Lab Lecture}

With only 3 hours to perform the practical portion of the lab, pre-lab preparation allows students to have a relatively good idea what they will be required to do prior to showing up in the lab.
The background lecture is based on information extracted from the original lab handout prepared by Dr. Eric Salt, Professor of Electrical Engineering.

The content of the lecture included:

Overview of some of the applications of microprocessors that we see in our daily lives.

Overview of the microprocessor board - see Figure 8.

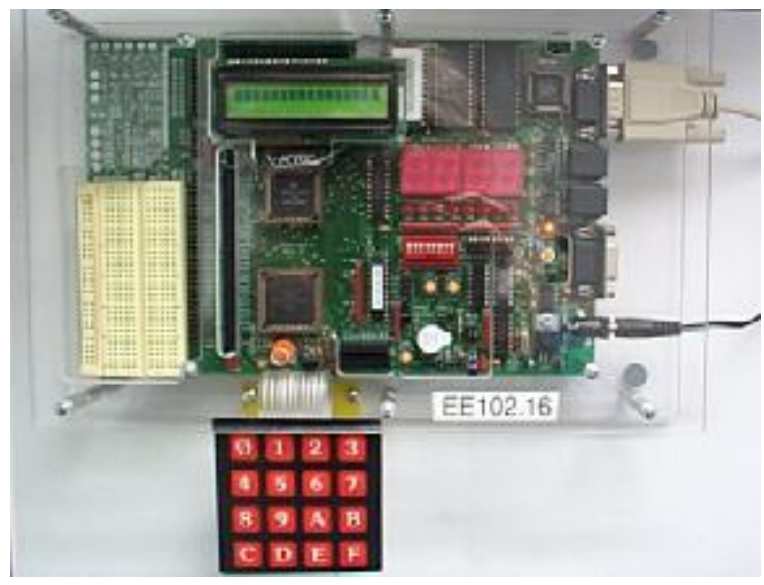

Figure 8 - Microprocessor board

An initial overview of the steps required to program the microprocessor. In an effort to make the lab run more smoothly, and to allow lab personnel to focus on real problems rather than procedural ones, it was made abundantly clear that students had to know the procedure. Even so, a number of lab problems were still traced back to this problem.

Write program in text editor/assembler program Connect microprocessor to PC (serial cable)

Run the assembler to produce executable code

Rest the microprocessor to run the monitor

Download the executable code via the monitor

Command the monitor to run executable code

Demonstration of downloading and running a program

Syntax (format) of comments / labels / instructions. Syntax errors are by far the most common that the students make in the lab, and one of the most frustrating. Reinforcing the importance of syntax, and the required format, are aimed at reducing the chance that students will get sidetracked with a syntax error.

Typical programming errors and messages. Notice was given that the lab starts with a series of intentionally introduced errors to familiarize students with typical errors and the accompanying error messages. The importance of observing the line number was emphasized. The initial onus for troubleshooting is thus put on the students, leaving 
the faculty/TAs to focus on more troublesome logic or procedural problems.

, Description of keyless garage door opener. Discussed previously

- Manually editing a combination.

-Writing a routine to change the combination. An algorithm is required to change the combination. In this case it was simply to add 4 to the previous combination.

- Creating an alternate combination. The method was introduced for creating an alternate combination by copying the existing combination code and modifying it. The most complex portion of the lab was to modify the main program to CORRECTLY accept both combinations. The function of the Main program, including the 3 key steps involved in testing the combination, was explained (only 16 lines, 3 of which were labels), and it was strongly suggested that close attention should be paid to the details of this part of the presentation so that they could perform the design portion of the lab. A solution was alluded to, and students were coached through the process of modifying the main program.

, Description of the hotel room door lock. Already discussed.

, Designing/implementing the hotel lock. When a new occupant checks in, both combinations must change. The next-occupant combination becomes the current-occupant combination, and a NEW nextoccupant combination is generated using the newcombination algorithm. It was discussed that there are several ways of implementing this, the easiest of which (which is not intuitively obvious, but was shown) is to run the new-combination function on both of the previous combinations, and to do it whenever a new-occupant combination is used.

\subsection{Lab Testing / Handout / Logistics}

The final steps prior to the lab were to ensure an accurate and error-free lab and handout, and ensuring the lab facility was set up to accommodate the lab groups and that lab personnel were ready to troubleshoot.

\subsubsection{Testing / Debugging of Lab and Handout}

The initial draft of the lab was tested using the first revision of the lab and handout. Some sections were modified to make corrections and enhanced clarity. A lack of ability to debug code, and the lack of time to teach a debugger resulted in a block of code being developed for debugging purposes. Two reserved combinations, when entered, would display the contents of the combination and the alternate combination. This very simple process allowed the examination of the only 2 variables that are changed by the program.

\subsubsection{Presentation of Handout}

The initial handout was 20 pages in length. Discussion with the faculty teaching the class resulted in a separation of the initial lab into a Lab lecture (described earlier) and an optional condensed lab procedure handout ( 9 pages). In the post-lab survey of students, who were provided the option of using either format, 4 out of 5 said they preferred separate theory/lab handout, versus the larger integrated one.

\subsubsection{Lab Logistics}

Students were divided into groups of 4 , with one microprocessor board per group. Nearly 400 students were handled over 5 lab periods, with two computer labs of 32 and 45 stations respectively per lab period.

One faculty member and one TA were assigned per lab period, so each was assigned to a different lab. Students were guided through the initial setup as a group, to minimize problems. Due to the well prepared lab handout, students worked relatively independently, and typical waiting times to get access to faculty/TA was only a matter of minutes.

\subsection{Summary and Feedback}

Implementation of this lab again went smoothly, and feedback from students was very positive. A number of key components are responsible for the success.

\subsubsection{Lab Design}

The keys to the successful design were:

- providing a good starting point, where a lot of the difficult application is already done

- providing the correct amount of background theory - enough to be usable, but not so much as to overwhelm

- strongly encouraging students to pay attention to the theory lecture

- testing the lab to ensure it was error free

- providing a lab overview prior to the lab period

- preparing students to troubleshoot their own errors

- designing a very strong lab handout

\subsubsection{Lab Communication}

The lab was divided into separate theory and lab instructions, which was later determined to be preferable by the students.

\subsubsection{Training of Lab personnel}

All lab personnel, including faculty and TAs, completed the lab well before the lab week, so that they could provide additional feedback and be prepared for the lab 
4.5.4 Feedback

A voluntary, anonymous survey was conducted, and well over 200 responses were received.

Approximately 9 out of 10 students that responded to the survey:

enjoyed the hands-on aspect of the lab

found the lab to be realistic and practical, and

felt that it gave them a better understanding of Electrical/Computer Engineering

felt that the Lab Overview lecture was required to understand the lab

Approximately 4 out of 5 students that responded to the survey:

felt that the Introduction to Digital Logic lecture was required to understand the lab

preferred the format of a separate lab lecture, and a condensed lab handout, versus a single combined document.

The responses show that the lab was overwhelmingly positively received.

\section{Conclusions}

The three methods presented for development of introductory design labs all proved to be very successful. Some common themes emerged in all of the methods. Sections 5.1 through 5.4 discuss common themes, while 5.5 on discuss other specific themes.

\subsection{Controlling Scope and Degrees of Freedom}

Relatively sophisticated or advanced concepts can be covered in design labs, if the problem is presented in a partially solved state, or if it is constrained well enough that limited background is not a limiting factor.

\subsection{High Quality Handout}

It is critical to have error-free and well designed lab handout. Any missing, unclear or confusing information, or an unintentional problem not core to the design lab, can torpedo an otherwise excellent lab.

\subsection{Correct Amount - Background Information} Great care must be taken to provide enough information to be useful, without overwhelming. The material provided must be focused, and the background preparation must be optimized.

\subsection{Training of Lab personnel}

A well planned lab handout should minimize the requirement for troubleshooting help or questions from students, however, the lab personnel has to be prepared for the lab. A dry-run by Faculty and
Teaching Assistants can be an excellent Quality Assurance process.

\subsection{Selecting a Partner - Industry Projects}

Good industry partners are ones with a connection to the college. Graduates of the college, or someone with ties to the college often work best because they are receptive and motivated. A forum such as this CDEN conference is an excellent opportunity to make networking contacts.

\subsection{Obtaining Design Data - Industry Projects}

Real design data is excellent for design labs. Unfortunately, it's often proprietary in industry. An incentive or workaround is to modify or sanitize data so that any proprietary data is protected. Peripheral data should be made available for reference in handouts, or on the web.

\subsection{Summary}

All of the methods investigated for developing Introductory Design labs were very successful as measured by the student feedback. Typical agreements with the achievement of goals were in the range of 9 out of 10 students.

\section{References}

[1] Eide, A.R., D.J. Jenison, L.H. Mashaw, and L.L. Northrup, "Engineering Fundamentals and Problem Solving - Fourth Edition", McGraw Hill, ISBN 0-07243027-3, pp. 80-81

[2] T.C. Muench, "Successfully Designing an Exciting, Hands-On, Practice-based Lab For Introductory Engineering Design", Integrating Practice Into Engineering Education, Dearborn, MI, October 2004

[3] Canadian Light Source - Synchrotron, Backgrounders and Quick Facts. Retrieved June 1, 2005 from http://www.lightsource.ca/media/quickfacts.php

[4] Canadian Light Source - Synchrotron, Component Specification-M1 Mask, Retrieved June 1, 2005 from http://www.engr.usask.ca/classes/GE/120

[5] C.D. Hawkes, "Casing Design Program for a Deep Oil Well", Unpublished laboratory handout - GE120, University of Saskatchewan, 2005.

[6] Bourgoyne Jr., A.T., Millheim, K.K., Chenevert, M.E. and Young Jr., F.S., Applied Drilling Engineering, Society of Petroleum Engineers, Richardson, Texas, 1991, $502 \mathrm{p}$

[7] JE. Salt, "GE 120 Laboratory for Electrical Engineering", Unpublished laboratory handout GE120, University of Saskatchewan, 2005.

[8] HC. Wood, "Introduction to Digital Computing", Unpublished lecture notes - GE120, University of Saskatchewan, 2005. 